\title{
Magnetically Stabilized Order II: Critical states and algebraically ordered nematic spin liquids in one-dimensional optical lattices
}

\author{
Hui Zhai ${ }^{\dagger}$ and Fei Zhou ${ }^{\dagger \dagger}$ \\ † Center for Advanced Study, Tsinghua University, Beijing, People's Republic of China and \\ ${ }^{\dagger}$ Department of Physics and Astronomy, University of British Columbia, \\ 6224 Agricultural Road, Vancouver, British Columbia, Canada, V6T $1 Z 1$
}

(Dated: December 22, 2018)

\begin{abstract}
We investigate the Zeeman-field-driven quantum phase transitions between singlet spin liquids and algebraically ordered $O(2)$ nematic spin liquids of spin-one bosons in one-dimensional optical lattices. We find that the critical behavior is characterized by condensation of hardcore bosons instead of ideal magnons in high- dimensional lattices. Critical exponents are strongly renormalized by hardcore interactions and critical states are equivalent to the free Fermion model up to the Friedel oscillations. We also find that the algebraically ordered nematic spin liquids close to critical points are fully characterized by the Luttinger-liquid dynamics with Luttinger-liquid parameters magnetically tunable. The Bethe Ansatz solution has been applied to determine the critical magnetization and nematic correlations.

PACS number: 03.75.Mn, 05.30.Jp, 75.10.Jm.
\end{abstract}

\section{INTRODUCTION}

It has been well known that spontaneous (continous) symmetry breaking states usually support gapless Goldstone modes which give arise various quantum fluctuations of local order parameters ${ }^{1,2.3 .4}$. In one dimension at zero temperature, long wave length quantum fluctuations can be overwhelming and extremely harmful to the establishment of long range order. Depending on details of energetics and topology of order parameter manifolds, quantum fluctuations might result in complete symmetry restoring by driving the states away from the spontaneous symmetry breaking solutions, or lead to algebraically long range order instead of the conventional offdiagonal long range order in high-dimensional systems.

For $O(3)$ nematic states of spin-one bosons in optical lattices (either condensates or Mott states) studied recently in quite a few articles 5 ,6.7.8.9.10.11.12, indeed in onedimensional lattices the symmetry is completely restored and resultant states are rotationally invariant. Unlike high-dimensional nematic states, spin excitations in these states are fully gapped as a result of symmetry restoring.

From the point of view of $(1+1) d$ Euclidean space, one can also attribute, at least partially, the disruption of long range order to the proliferation of Meron- or Skyrmion-like topological instantons. This fact suggests that the topology of vacuum manifold (mean field) should play an important role in maintaining or destroying long range order.

For instance, when a magnetic field is applied to $O(3)$ nematic states, the easy axis of the nematic order parameter tensor is pinned in a plane perpendicular to the external field. This lows the symmetry of nematic order and the $O(3)$ symmetry is reduced to $O(2)$ one, i.e. the state becomes a planar nematic state. For nematic condensates this mechanism leads to a vacuum manifold of $\left[S^{1} \times S^{1}\right] / Z_{2}$ instead of $\left[S^{1} \times S^{2}\right] / Z_{2}$ in the absence of magnetic fields.

The above observation then implies that in onedimension in the presence of external magnetic fields long wave length fluctuations should not completely disrupt nematic ordering in a plane perpendicular to the external fields as pointed out in a previous article ${ }^{5}$. Instead one should expect an algebraically ordered nematic spin liquid. In this paper we are going to investigate this possibility from a very different starting point and develop a theory which fully and quantitatively characterizes fieldinduced algebraically ordered nematic spin liquids in onedimensional optical lattices.

A closely related subject, magnetically stabilized nematic order in three-dimensional bipartite lattices, has also been studied recently ${ }^{12}$. It was found that the stabilization of nematic order can be studied via a mapping to the ferromagnetic $X X Z$ model and the critical phenomenon is identical to condensation of ideal bosonic magnons. Away from critical fields the nematically ordered states are described by weakly interacting dilute magnons.

We are going to employ the previously developed mapping between the low energy Hilbert space close to critical fields and the ferromagnetic $S=1 / 2 X X Z$ model to further analyze one-dimensional field-induced algebraically ordered spin nematic liquids. Though the general approach employed below is similar to that developed for three-dimensional lattices, the unique feature of dilute magnons in the one-dimension limit makes the current situation distinct and physics qualitatively different from that discussed in the previous article. In fact, it is the distinct properties of fermionized dilute magnons which lead to anomalous critical exponents as well as fascinating algebraically ordered nematic liquids discussed below.

In Sect. II, we are going to review some basic results of the mapping between the field-driven quantum phase transitions (between spin singlet Mott states and nematic Mott states) and the ferromagnetic $S=1 / 2$ 
$X X Z$ model. We further demonstrate the linear relation between the pseudo-spin operators in the $X X Z$ model and nematic tensor order parameter operators. In Sect. III, we study the critical behavior of these quantum phase transitions in the Holstein-Primakov boson model, and point out that one-dimensional quantum fluctuations strongly renormalize the critical exponents. Unlike in three-dimensional lattices where the critical point is fully characterized by ideal bosons (noninteracting), in one-dimensional lattices the critical point is characterized by hardcore bosons with an infinitely large interaction constant. And we believe our evaluation of the critical exponent is exact.

In Sec. IV, we further investigate the critical point physics using the Jordan-Wigner's fermionic representation. We obtain an identical critical exponent as in Sec. III. We argue that the critical point is also fully characterized by the free Fermion model; however the Friedel oscillations, one of the basic aspects of Fermions, do not appear in the correlation function of nematic order operators.

In Sec. V, we further study the one-dimensional algebraically ordered nematic spin liquids close to critical fields using a strongly interacting boson model. We employ the technique of bosonization to evaluate various correlation functions in the nematic spin liquids and argue that in general these liquids, up to the Friedel oscillations are equivalent to the Luttinger liquids of Fermions. In Sec. VI, we conclude our studies and discuss some consequences of these theoretical findings.

\section{THE EFFECTIVE THEORY CLOSE TO CRITICAL POINTS: THE FERROMAGNETIC $S=1 / 2 X X Z$ MODEL}

We introduce $\psi_{k \alpha}^{\dagger}\left(\psi_{k \alpha}\right), \alpha=x, y, z$ as the creation (annihilation) operators for a spin-one particle in three orthogonal states at site $k$. The effective Hamiltonian for Mott states of spin-one bosons with antiferromagnetic interactions in optical lattices in an external field can be conveniently expressed as s.9.10.11.12, $^{7.12}$

$$
\begin{aligned}
& H=E_{s} \sum_{k} \hat{\mathbf{S}}_{k}^{2}-\sum_{k} \hat{\mathbf{S}}_{k z} H_{z} \\
& -J_{e x} \sum_{\langle k l\rangle}\left(\hat{Q}_{\alpha \beta}(k) \hat{Q}_{\beta \alpha}(l)+h . c .\right) ; \\
& \hat{Q}_{\alpha \beta}(k)=\psi_{k \alpha}^{\dagger} \psi_{k \beta}-\frac{1}{3} \delta_{\alpha \beta} \psi_{k \gamma}^{\dagger} \psi_{k \gamma} .
\end{aligned}
$$

Here $E_{s}$ is the "bare" spin gap studied in Ref. (7) and $J_{e x}$ is the strength of exchange interaction. $\hat{\mathbf{S}}_{k \alpha}=$ $-i \epsilon_{\alpha \beta \gamma} \psi_{k \alpha}^{\dagger} \psi_{k \beta}$ is the total spin operator for lattice site k. $\hat{Q}_{\alpha \beta}(k)$ is a bilinear tensor operator, the expectation of which yields the nematic order parameter. Finally, the sum over $\langle k l\rangle$ represents a sum over all neighboring sites. And again we are interested in the case with an even number of particles per lattice site, though all conclusions are qualitatively correct for an odd number of particles per site.

In high-dimensional lattices, when the external field is absent and $\eta=J_{e x} / E_{s}$ is smaller than a critical value $\eta_{c}$, the ground state is a spin singlet Mott state for an even number of particles per site $(N)$. And when $\eta$ is larger than $\eta_{c}$, the ground state is a nematic Mott state. The critical value $\eta_{c}$ has been calculated in various limits and turns out to be of order of unity 10 .

In one-dimensional lattices, in the absence of external magnetic fields the ground state is always a spin singlet one and the mean field solution with nematic ordering is unstable. When $J_{e x}$ is set to be zero, at each lattice site the lowest energy state is a spin singlet one $(S=0)$ and the first excited states are fivefold degenerate $S=2$ states. The ground state in this case is simply a product of spin singlets at each lattice.

When an external field is applied along the $z$-axis, one of the five-fold degenerate $S=2$ states approaches the spin singlet and level crossing takes place. Generally speaking close to the level crossing points at each site, two states $\mid S, S_{z}>$ and $\mid S+2, S_{z}+2>$ become degenerate; the on-site low energy Hilbert space is spanned by the two states involved in the level crossing.

It is therefore convenient to express the effective low energy Hamiltonian in this pseudo-spin subspace. Consider the crossing between states $\mid 0,0>$ and $\mid 2,2>$. If one introduces the on-site pseudo spin Hilbert space $\mathcal{S}_{k}$ which consists of two states,

$$
|\uparrow\rangle=\left|S=2, S_{z}=2\right\rangle,|\downarrow\rangle=\left|S=0, S_{z}=0\right\rangle,
$$

then the truncated Hilbert space for the whole lattice $\mathcal{S}_{T}$ is the product of pseudo spin Hilbert space $\mathcal{S}_{k}$ at each site $k$, i.e. $\mathcal{S}_{T}=\mathcal{S}_{1} \otimes \mathcal{S}_{2} \otimes \ldots \otimes \mathcal{S}_{k} \otimes \ldots$

Defining $|\uparrow\rangle$ and $|\downarrow\rangle$ to be two eigenstates of Paulimatrix $\sigma_{z}, \sigma_{z}|\uparrow\rangle=|\uparrow\rangle, \sigma_{z}|\downarrow\rangle=-|\downarrow\rangle$, one obtains the effective Hamiltonian which is represented by the ferromagnetic $X X Z$ model in an effective external field along the $z$-direction ${ }^{12}$,

$$
\frac{H_{X X Z}}{\epsilon_{0} J_{e x}}=-\sum_{\langle k l\rangle} \sigma_{k \alpha} \sigma_{l \alpha}-\beta \sum_{\langle k l\rangle} \sigma_{k z} \sigma_{l z}-h_{z} \sum_{k} \sigma_{k z} .
$$

Eq.(3) holds when the truncation is valid, which requires that $J_{e x}$ should be much less than $E_{s}$. Here $\epsilon_{0}, \beta$ and $h_{z}$ were calculated in Ref.(12).

The Hamiltonian in Eq. (3) has an $O(2)$ invariance in the $x y$-plane corresponding to the $O(2)$ rotational invariance in the microscopic Hamiltonian in Eq. (II) when the external magnetic fields are applied along the $z$-direction. This $O(2)$ invariance represents the $O(2)$ nematic symmetry we are going to examine. Furthermore, in general the truncation can be applied in the vicinities of all critical points where level crossings between $\left|S, S_{z}=S\right\rangle$ and $\left|S+2, S_{z}=S+2\right\rangle$ occur, $S+2 \leq N$. 
The main issue dealt in this work is the induced spontaneous symmetry breaking in the presence of external magnetic fields. Particularly we are interested in the breaking of $O(2)$ rotational symmetry in the $x y$-plane when a field is applied along the $z$-axis. Unfortunately all operators that are linear in $\psi_{\alpha}^{\dagger}$, or $\psi_{\alpha}$ vanish identically in Mott states regardless whether the rotational symmetry is broken or not; so linear operators fail to distinguish two phases which interest us.

The rank-two-tensor order parameter introduced on the other hand vanishes only in rotationally invariant spin singlet states and develops nontrivial structures once states break the $O(2)$ symmetry. For this reason we introduce the projected nematic order parameter $\hat{Q}_{\alpha \beta}^{P}(\alpha, \beta=x, y, z)$ which is designed to signify the $O(2)$ symmetry breaking in the $x y$ plane as follows

$$
\hat{Q}_{\alpha \beta}^{P}=\hat{Q}_{\alpha \beta}-\left(\hat{Q}_{\alpha^{\prime} \beta^{\prime}} \Pi_{\beta^{\prime} \alpha^{\prime}}^{1}\right) \Pi_{\alpha \beta}^{1}-\left(\hat{Q}_{\alpha^{\prime} \beta^{\prime}} \Pi_{\beta^{\prime} \alpha^{\prime}}^{2}\right) \Pi_{\alpha \beta}^{2} .
$$

Two constant tensors we would like to project away are defined as ${ }^{12}$

$$
\Pi_{\alpha \beta}^{1}=\frac{1}{\sqrt{2}} i \epsilon_{\alpha \beta \gamma} \mathbf{s}_{\gamma}, \Pi_{\alpha \beta}^{2}=\frac{3}{\sqrt{6}}\left(\mathbf{s}_{\alpha} \mathbf{s}_{\beta}-\frac{1}{3} \delta_{\alpha \beta}\right) ;
$$

and $\mathbf{s}$ is the unit vector along the direction of spin polarization which in our case is along the $z$-axis. It is evident following the discussions in Ref.(12) that the vacuum manifold (mean field) of field-induced spin nematic states is $S^{1} / Z_{2}$, identical to that of the standard planar nematic order parameter.

In the pseudo spin subspace, the above operator can be expressed in terms of the Pauli matrices $\sigma^{ \pm}$,

$$
\hat{Q}_{\alpha \beta}^{P}=\sigma^{+} \Gamma_{\alpha \beta}+\sigma^{-} \Gamma_{\alpha \beta}^{\dagger}, \alpha, \beta=x, y, z ;
$$

and $\Gamma_{\alpha \beta}$ is a constant symmetric traceless $3 \times 3$ matrix. The detailed form of the $\Gamma$-matrix depends on the number of particles per site as well as the level crossings involved. One can find more discussions on the calculation of the $\Gamma$-matrix in Appendix A.

For instance for 2-particles per lattice site, one finds

$$
\Gamma_{\alpha \beta}=\frac{1}{\sqrt{3}}\left(\begin{array}{ccc}
1 & -i & 0 \\
-i & -1 & 0 \\
0 & 0 & 0
\end{array}\right)
$$

and for four-particles per site, one obtains

$$
\Gamma_{\alpha \beta}=\sqrt{\frac{14}{15}}\left(\begin{array}{ccc}
1 & -i & 0 \\
-i & -1 & 0 \\
0 & 0 & 0
\end{array}\right) .
$$

It is more convenient to further truncate the projected nematic tensor operator $\hat{Q}_{\alpha \beta}^{P}$ in the $x y$-plane which interests us by only keeping the elements with $\alpha, \beta=x, y$. The truncated operator then has the following structure

$$
\hat{Q}_{\alpha \beta}^{P x y}=\sigma^{+} T_{\alpha \beta}+\sigma^{-} T_{\alpha \beta}^{\dagger}, \alpha, \beta=x, y ;
$$

and $T_{\alpha \beta}$ is a constant symmetric traceless $2 \times 2$ matrix obtained by truncating the $\Gamma$-matrix. For 2 -particles per lattice site,

$$
T_{\alpha \beta}=\frac{1}{\sqrt{3}}\left(\begin{array}{cc}
1 & -i \\
-i & -1
\end{array}\right) .
$$

Finally, the magnetization can also be expressed in terms of the pseudo spin operators. For instance, the $z$-component is simply proportional to $\sigma_{z}$. Following the definition of the pseudo spin operators, one finds that

$$
M_{k z}=\sigma_{k z}+1 .
$$

(Here we have set the Bohr magneton as one). The relation between the nematic tensor operator and Pauli matrices, together with the effective Hamiltonian allow us to investigate various nematic spin correlations in terms of the $X X Z$ model.

We also would like to point out that the subspace spanned by pseudo spin states is actually a polarization (in the $x y$ plane) free subspace. That is

$$
<\mathbf{S}_{\alpha}>=0, \alpha=x, y .
$$

The absence of polarization (in the $x y$ plane) is a usual property of a planar spin nematic state.

\section{CRITICAL STATES AS 1D HARDCORE-BOSON GASES}

As demonstrated in a previous work, the field-driven quantum phase transitions between spin singlet and spin nematic Mott states can be studied in terms of the condensation of spin-two magnons in spin singlet Mott states ${ }^{12}$. To carry out discussions along this line, it is rather convenient to introduce the Holstein-Primakov representation for the XXZ ferromagnetic spin model. In this representation, all spin operators are expressed in terms of Holstein-Primakov bosons,

$$
\begin{aligned}
\sigma^{-} & =\left(\sqrt{1-c^{\dagger} c}\right) c \\
\sigma^{+} & =c^{\dagger} \sqrt{1-c^{\dagger} c} \\
\sigma_{z} & =2 c^{\dagger} c-1
\end{aligned}
$$

$c^{\dagger}(c)$ is the creation (annihilation) operator of bosons satisfying the usual bosonic commutation relations $\left[c, c^{\dagger}\right]=$ 1 ; and the raising and lowering operators are defined as $\sigma^{+}=\left(\sigma_{x}+i \sigma_{y}\right) / 2, \sigma^{-}=\left(\sigma_{x}-i \sigma_{y}\right) / 2$. One can furthermore verify that $\left[\sigma_{\alpha}, \sigma_{\beta}\right]=i 2 \epsilon_{\alpha \beta \gamma} \sigma^{\gamma}$ and $\sigma \cdot \sigma=3$. The Hamiltonian of the XXZ model then transforms into 12 


$$
\begin{aligned}
\frac{H_{X X Z}}{\epsilon_{0} J_{e x}}= & -2 \sum_{\langle k l\rangle} \sqrt{\left[1-c_{k}^{\dagger} c_{k}\right]} c_{k} c_{l}^{\dagger} \sqrt{1-c_{l}^{\dagger} c_{l}} \\
& -2 \sum_{\langle k l\rangle} c_{k}^{\dagger} \sqrt{\left(1-c_{k}^{\dagger} c_{k}\right)\left(1-c_{l}^{\dagger} c_{l}\right)} c_{l} \\
& -4(1+\beta) \sum_{\langle k l\rangle} c_{k}^{\dagger} c_{k} c_{l}^{\dagger} c_{l} \\
& +\left(4(1+\beta)-2 h_{z}\right) \sum_{k} c_{k}^{\dagger} c_{k} ;
\end{aligned}
$$

Previous calculations in Ref.(12) show that $\beta$ is always negative for interacting spin-one bosons under consideration.

In the dilute gas limit which interests us, the number of Holstein-Primakov bosons per lattice site is much less than one i.e. $n_{c}=\left\langle c_{k}^{\dagger} c_{k}\right\rangle \ll 1$. For this reason, one can expand the nonlinear operators of $\sigma^{ \pm}$in terms of $n_{c}$. Indeed, the resultant many-body Hamiltonian up to the second order of $n_{c}$ is

$$
\begin{aligned}
& \frac{H_{X X Z}}{\epsilon_{0} J_{e x}}=\sum_{q}\left[4-4 \cos q+4 \beta-2 h_{z}\right] c_{q}^{\dagger} c_{q} \\
& +\frac{1}{V_{T}} \sum_{q_{1}, q_{2}, q} V_{B}(q) c_{q_{1}+q}^{\dagger} c_{q_{2}-q}^{\dagger} c_{q_{1}} c_{q_{2}}, \\
& V_{B}(q)=-4 \beta \cos q+O\left(\rho^{2}\right) .
\end{aligned}
$$

The lattice constant $a$ has been set as one. And at small $q$ the dimensionless mass of bosons $m$ is $1 / 4$.

The Hamiltonian in Eq.(15), or Eq.(14) in fact describes bosons interacting with short range interactions. The bare two-body repulsive interaction potential $V_{B}(k l)$ when two particles are in two neighboring sites $k$ and $l=k \pm 1$ is $-4 \beta$ (in units of $\epsilon_{0} J_{e x}$, and $-1<\beta<0$ ) i.e.,

$$
V_{B}(k l)=-4 \beta \delta_{k \pm 1, l} .
$$

Therefore, the $q$-dependent two-body interaction constant becomes $-4 \beta$ as $q$ approaches zero.

Following Eq. 151), when $h_{z}<h_{c}=2 \beta$, all magnon excitations are fully gapped with the energy gap being $2\left(h_{c}-h_{z}\right)$. The ground state therefore is a vacuum of the bosonic field or a spin singlet Mott state; at $h_{z}=$ $h_{c}=2 \beta$, excitations with $q=0$ become gapless signifying a quantum phase transition between spin singlet states and nematic states. When $h_{z}>h_{c}$, magnons appear in the ground state and the density is determined by the following standard equilibrium condition

$$
2 h_{z}-2 h_{c}=\mu(\beta, \rho) .
$$

Here $\mu$ is the chemical potential depending on the density $\rho$ and the parameter $\beta$. Therefore nematic Mott states close to critical points are effectively described by dilute interacting bosons (spinless). Eq.(17) indicates that close to critical points $h_{z}-h_{c}$ approaches zero and bosons are in the dilute limit.

A similar set of equations have been derived and studied in Ref.(12). In high-dimensional lattices, one can easily apply the results on weakly interacting bosons to analyze field-induced nematic Mott states at and close to critical points. This has been achieved in a previous work and the relation between the development of nematic order and condensation of magnons has been fully investigated 12 . The chemical potential of a weakly interacting magnon condensate with interaction given in Eq.(16) is simply $-8 \beta \rho$. Following Eq.(17), this leads to the magnon density as well as the magnetization close to the critical field.

However, in one-dimensional lattices with $V_{T}$ lattice sites and a given number of bosons, the single-particle zero point motion energy scales as $V_{T}^{-2}$ while the interaction energy per particle scales as $V_{T}^{-1}$. As a result, the dimensionless coupling constant $\gamma$ which is defined as the ratio between the interaction energy per particle $(-4 \rho \beta)$ and the kinetic energy of a particle confined within a length scale $\rho^{-1}\left(\rho^{2}\right)$ is inversely proportional to $\rho$, the number of particles per lattice site,

$$
\gamma=-\frac{4 \beta}{\rho}
$$

and $\gamma$ diverges as $\rho$ approaches zero in the dilute limit close to critical points. One-dimensional dilute bosons with interactions are equivalent to hardcore bosons as the density $\rho$ is taken to be zero and $\gamma$ becomes infinite. The critical states in one dimension are distinctly different from free bosons.

As pointed out by Girardeau ${ }^{18}$, the ground state wave functions of hardcore bosons $(\gamma=\infty)$ in the continuum limit can be expressed as the absolute value of the Slater determinant of free fermions. Liniger and Lieb further investigated cases when $\gamma$ varies from zero to infinity using the Bethe Ansatz method ${ }^{19}$. In addition to confirming the solution in the impenetrable hardcore boson limit obtained by Girardeau, they also pointed out the important functional relation between the two-body scattering phase shift $\theta\left(q_{1}, q_{2}\right)$ (see more discussions in Appendix B) and the chemical potential of interacting bosons for an arbitrary $\gamma$. Especially, at large $\gamma$ limit the chemical potential of dilute bosons approaches the value of the chemical potential of free fermions with an identical density.

To understand the development of magnetization close to critical points, we outline the calculation of the chemical potential of the Holsetin-Primakov bosons in the dilute limit. We first notice that in the continuum limit the two-body interaction in Eq.(16) becomes delta-like with strength being equal to $-4 \beta$. Following Ref 19 (see more discussions in Appendix B), we find that the chemical potential for bosons with the two-body interaction 
$-4 \beta \delta\left(x-x^{\prime}\right)$ is determined by the following set of equations

$$
\begin{aligned}
& \int d q n(q)=\rho, \\
& \frac{N}{\rho} \int d q n(q) \frac{q^{2}}{2 m}=E_{0}, \\
& -8 \beta \int d p \frac{n(p)}{16 \beta^{2}+(p-q)^{2}}=2 \pi n(q)-1 .
\end{aligned}
$$

Here $n(q)$ is the distribution function of qausimomentum; the dimensionless mass $m$ is equal to $1 / 4$. $N$ is the total number of particles. The first two equations above are the definitions of the number density of particles $\rho$ and the total energy $E_{0}$ of the system. The last one in Eq.(19) follows the Bethe Ansatz solution. This set of equations have been studied in Ref 19 and for the convenience of readers we present the derivation in Appendix B. The Bethe Ansatz solution for the $X X Z$ spin model was also obtained by Yang and Yang 20 .

The discussion in Appendix B indicates that the solution of Eq. (19) should yield the following chemical potential in the dilute limit

$$
\mu=\frac{\partial E_{0}}{\partial N}=\frac{2 \pi^{2}}{3} \frac{3 \gamma^{3}+2 \gamma^{2}}{(\gamma+2)^{3}} \rho^{2} .
$$

And $\gamma$ is defined in Eq.(18). In the presence of external fields, in equilibrium the chemical potential has to be equal to $2 h_{z}-2 h_{c}(>0)$ as suggested in Eq.(17). This leads to the equilibrium density of strongly interacting magnons close to critical points where the dimensionless interaction constant $\gamma$ diverges as indicated in Eq.(18),

$$
\rho\left(h_{z}\right)=\frac{1}{\pi} \sqrt{h_{z}-h_{c}}+\ldots
$$

Taking into account Eq.(11), one obtains

$$
M_{z}=2 c_{k}^{\dagger} c_{k}
$$

and derives the magnetization close to the critical point as

$$
M_{z}=\frac{2}{\pi} \sqrt{h_{z}-h_{c}}
$$

We should emphasize here that because of the large dimensionless coupling constant of dilute bosons in onedimensional lattices, the chemical potential is a quadratic function of the density of bosons instead of a linear function as in the weakly interacting limit. As a result, in one-dimensional lattices the critical exponent in the quantum phase transitions between the nematic and spin singlet Mott states is renormalized to be one-half of the critical exponent found for high-dimensional lattices (See Ref 12).

Slightly away from critical points, we also obtain the corrections to the magnon density as well as the magnetization by expanding Eq.(20) in terms of a large but finite $\gamma$. In the dilute limit, the chemical potential approximately is

$$
\mu \simeq \frac{2 \pi^{2}}{3}\left[3 \rho^{2}+\frac{4}{\beta} \rho^{3}\right] .
$$

The leading correction to the magnetization $M_{z}$ in Eq. (23) is

$$
\delta M_{z}=\frac{2}{3 \pi^{2}|\beta|}\left(h_{z}-h_{c}\right)+\cdots
$$

which becomes significant when $h_{z}-h_{c}$ becomes comparable to $\beta^{2}$.

In Sec. IV, we are going to further examine the critical states using the Jordan-Wigner Fermionic representation. In Sec. V, we apply the general bosonization approach for one-dimensional fluid to the states close to the critical points. Finally we would like to mention that both bosonic and Majorana fermionic representation have been employed to study antiferromagnetic spin chains in an external field 13,14,15,16. Bethe Ansatz solutions of the XXZ model have also been used to calculate critical exponents in various models $\frac{17}{}$.

\section{CRITICAL STATES AS FREE-FERMION GASES}

Since the ground state wave functions of interacting bosons in the dilute limit, or equivalently hardcore bosons can be related to the Slater determinant of free Fermions, not surprisingly many aspects of the fixed point solutions reminisce free Fermions. It is certainly appealing to work with the fermionic representation (of Jordan-Wigner type) and find out to what extend the critical points can be characterized by the free Fermion model. In this section we are going to implement this idea.

In the Jordan-Wigner representation, spin-operators are expressed in terms of string operators defined by Fermions,

$$
\begin{aligned}
\sigma_{k}^{+} & =\exp \left(i \pi \sum_{l<k} f_{l}^{\dagger} f_{l}\right) f_{k}^{\dagger} \\
\sigma_{k}^{-} & =f_{k} \exp \left(-i \pi \sum_{l<k} f_{l}^{\dagger} f_{l}\right) \\
\sigma_{k z} & =2 f_{k}^{\dagger} f_{k}-1 .
\end{aligned}
$$

The string operator in the Wigner-Jordan form is oriented along the path starting at $-\infty$ and ending at $k-1$.

Substituting the expressions for spin operators, one rewrites the ferromagnetic XXZ model in the following form (see more discussions in Appendix C) 


$$
\begin{aligned}
& \frac{H_{X X Z}}{\epsilon J_{e x}}=-2 \sum_{<k l>}\left(f_{k}^{\dagger} f_{l}+f_{l}^{\dagger} f_{k}\right)+ \\
& +\left[4(1+\beta)-2 h_{z}\right] \sum_{k} f_{k}^{\dagger} f_{k} \\
& -4(1+\beta) \sum_{<k l>} f_{k}^{\dagger} f_{k} f_{l}^{\dagger} f_{l} .
\end{aligned}
$$

Eq.(27) shows that the ferromagnetic $X X Z$ model $(0>\beta>-1)$ is equivalent to attractively interacting Fermions. The bare two-particle attractive interaction $V_{F}(k l)$ is short ranged; the interaction is nonzero only when two particles occupy two neighboring sites and has a negative value of $-4(1+\beta)$, i.e.

$$
V_{F}(k l)=-4(1+\beta) \delta_{k \pm 1, l} .
$$

Obviously the bare potential vanishes only when $\beta=-1$.

However, because of the Pauli exclusion principle, it is well known that scattering between spinless Fermions is absent if the two-particle potential is truly $\delta$ - like. For a similar reason in a dilute Fermi gas the scattering is very weak if the two-body potential is short ranged. Therefore, in one-dimension for a given number of Fermions while the one-particle kinetic energy for Fermions is proportional to $V_{T}^{-2}$ as in the case of bosons, the interaction energy per Fermion is proportional to $V_{T}^{-3}$ instead of $V_{T}^{-1}$ as a result of the Pauli exclusion principle. It indicates that the dilute limit of Fermions is truly weakly interacting and the fixed point of free Fermions is stable. Indeed, in one-dimensional lattices, the dimensionless coupling constant scales as $\rho a$ in the dilute limit ( $a$ is the range of interaction which in our case is the lattice constant; it is set as one in this article).

Following the discussions in Appendix C, one obtains the following effective Hamiltonian

$$
\begin{aligned}
& \frac{H_{X X Z}}{\epsilon J_{e x}}=\sum_{q}\left[4 \beta-2 h_{z}+4-4 \cos q\right] f_{q}^{\dagger} f_{q} \\
& +\frac{1}{V_{T}} \sum_{q_{1}, q_{2}, q} V_{F}(q) f_{q_{1}-q}^{\dagger} f_{q_{1}} f_{q_{2}+q}^{\dagger} f_{q_{2}} ; \\
& V_{F}(q)=4(1+\beta)(\cos q-1)<0
\end{aligned}
$$

And again at the critical point $h_{c}=2 \beta$, the excitations with $q=0$ become unstable and the quantum phase transition occurs. When $h_{z}<h_{c}$, Fermionic excitations are gapped with an energy $2\left(h_{c}-h_{z}\right)$ and the ground state is a vacuum of Fermions representing the spin singlet Mott state. Slightly above the critical field, Fermions with small momenta have negative energies and we have a dilute interacting Fermi gas in the ground state representing nematic Mott states.

The second sum in Eq.(29) indicates that the forward scattering amplitude between right-movers or left-movers in one-dimension, i.e. $\left(r q_{F}, r q_{F}\right) \rightarrow\left(r q_{F}, r q_{F}\right)(r= \pm)$ is proportional to $q^{2} \sim 0$ ( $q$ denotes the transferred momentum during the scattering and $q_{F}$ is the Fermi momentum) and therefore vanishes in the long wave length limit. However the back scattering between right-movers and left-movers, $\left(r q_{F},-r q_{F}\right) \rightarrow\left(-r q_{F}, r q_{F}\right)$ involves a momentum transfer $q=2 q_{F}$; the scattering amplitude is proportional to $q_{F}^{2}$ and remains finite in the long wave length limit. As expected before, the dimensionaless coupling constant which is the ratio between the interaction energy $\epsilon(1+\beta) \rho$ and the kinetic energy $\epsilon$ should be proportional to

$$
\gamma_{F}=-(1+\beta) \rho
$$

Notice that the bare two-particle attractive interactions $-4(1+\beta)$ are nonzero for any system with $\beta \neq-1$ and can even be comparable to the Fermi energy. In principle, Fermions under consideration can be in the strongly attracting limit. However, the effective coupling constant which itself depends on the density of Fermions is always small in the extremely dilute limit. And it becomes precisely zero when critical points are approached and one anticipates that the Fermion density vanishes. Close to the critical points, we do have a collection of weakly interacting Fermions, a problem which can be easily handled. Of course when $\beta+1=0$, the bare two-body interactions become zero identically.

To summarize the result of this mapping, we would like to emphasize that all states along the triangular boundaries in the phase diagram in Fig.1 a) correspond to the free Fermions. The $O(3)$ symmetric tricritical point on the other hand is a free boson critical point. All states within the triangular boundaries are Luttinger liquids with varying densities and Luttinger liquid parameters. Finally states in the SSMI phase outside the triangular boundaries in Fig. 1c) are represented as the vacuums of Jordan-Wigner Fermions and the SPMI corresponds to a filling factor $N=1$ Fermionic Mott state (see Fig.1 for more discussions).

Therefore, the critical point can also be studied conveniently in the Fermionic representation. For instance, the chemical potential in this case is naturally proportional to the square of the Fermion density which is consistent with the results obtained in the bosonic representation. Taking into account that $m=1 / 4$, one obtains

$$
\mu_{F}=2 \pi^{2} \rho^{2}
$$

Along with the equilibrium condition for Fermi gases (which is identical to the condition for bosons in Eq.(17))

$$
\mu_{F}(\beta, \rho)=2\left(h_{z}-h_{c}\right),
$$

one obtains the Fermi density close to the critical point.

Eq.(11) and Eq.(26) further indicate that the magnetization is proportional to the Fermion number operator 


$$
M_{k z}=2 f_{k}^{\dagger} f_{k} .
$$

This again leads to Eq.(23).

Moreover, one can express nematic tensor operator in terms of the Fermion operators

$$
\hat{Q}_{\alpha \beta}^{P x y}=T_{\alpha \beta} \exp \left(i \pi \sum_{l<k} f_{l}^{\dagger} f_{l}\right) f_{k}^{\dagger}+\text { h.c. }
$$

In deriving it one has to take into account Eq.9)and Eq.(26), the mapping between the pseudo-spin operator and Fermion operators. Note the nematic operator has nonlocal dependence on the Fermion operators: it is a string operator of Fermions! This aspect of the tensor operator plays a very important role in the nematic correlations in 1D.

So we can use the free Fermion properties to calculate the nematic tensor correlators in 1D. Indeed, we find that the correlator appears to be clearly related to the free Fermion correlator,

$$
\begin{aligned}
& <\hat{Q}_{\alpha \beta}^{P x y}(k) \hat{Q}_{\alpha^{\prime} \beta^{\prime}}^{P x y}(l)>=T_{\alpha \beta} T_{\alpha^{\prime} \beta^{\prime}}^{\dagger} \\
& <f_{k} \exp \left(-i \pi \sum_{n<k} f_{n}^{\dagger} f_{n}\right) \exp \left(i \pi \sum_{m<l} f_{m}^{\dagger} f_{m}\right) f_{l}^{\dagger}>+c . c .
\end{aligned}
$$

Evaluating this correlator is tricky because a Fermion operator itself inexplicitly contains string operators and is correlated with the string operator. The consideration towards the end of Sec. V and in Appendix C implies that the contribution from the string operator in the JordanWigner representation yields precisely a sign oscillation in the Fermion wave function. So the appearance of the Jordan-Wigner phase factor in the nematic correlator ensures that such oscillations in the free Fermion correlator get cancelled out. Finally, standard calculations yield

$$
<\hat{Q}_{\alpha \beta}^{P x y}(k) \hat{Q}_{\alpha^{\prime} \beta^{\prime}}^{P x y}(l)>\sim T_{\alpha \beta} T_{\beta^{\prime} \alpha^{\prime}}^{\dagger} \frac{1}{|k-l|^{1 / \eta_{0}}},
$$

where the Friedel oscillations that appear in the free Fermion correlator are indeed absent in the nematic correlations (We have also neglected the higher harmonics). $\eta_{0}=2$ following the Luttinger liquid theory (see Appendix $\mathrm{D}$ ). In the next section, we are going to study the nematic states close to critical points using the bosonization technique.

\section{ALGEBRAICALLY ORDERED SPIN NEMATIC LIQUIDS CLOSE TO CRITICAL POINTS}

Following the discussion at the end of section IV and Appendix C, as far as the nematic correlations are concerned, we notice that the Fermion representation of

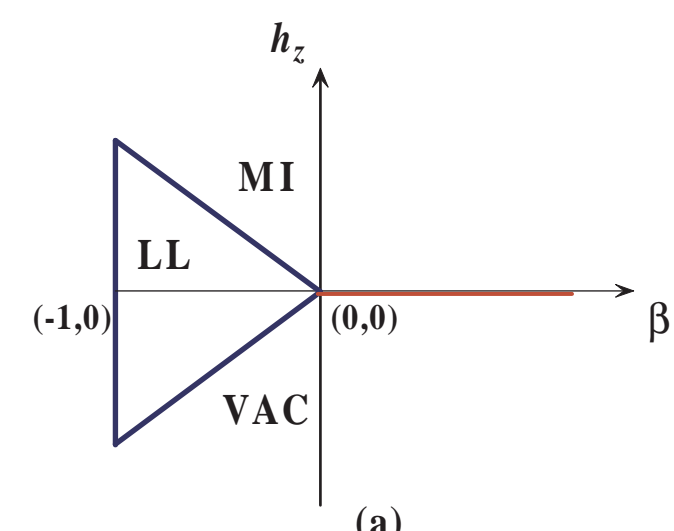

(a)

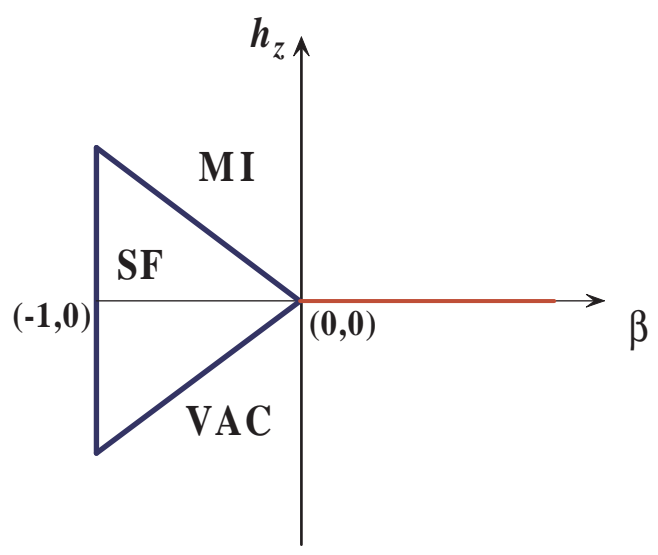

(b)

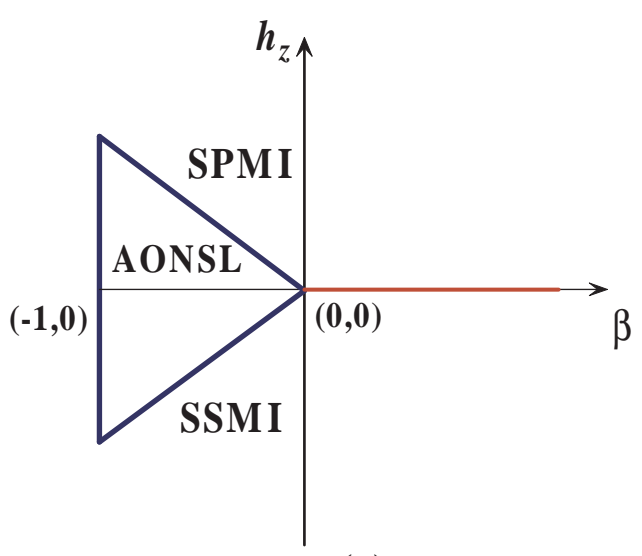

(c)

FIG. 1: (Color online) a) The phase diagram for the JordanWigner Fermions in the $h_{z^{-}} \beta$ plane $(\beta \in[-1, \infty])$. Inside the triangle are the Luttinger liquids (LL). Outside the triangle and $h>0[<0]$ is the Mott insulator (MI) [vacuum (VAC)] of spinless Fermions. Along the boundary of the triangle is the free Fermi gas. b) The phase diagram for the HolsteinPrimakov bosons (1D) in the $h_{z}-\beta$ plane. Inside the triangle is the superfluid (SF). Outside the triangle and $h>0[<0]$ is the Mott insulator (MI) [vacuum (VAC)] of bosons. Along the boundary of the triangle is the hardcore boson gas. c) The phase diagram for spin-one bosons in 1D optical lattices indicated by a) and b). Inside the triangle is the algebraically ordered nematic spin liquid (AONSL). Outside the triangle and $h>0[<0]$ is the spin polarized Mott insulator (SPMI) [spin singlet Mott insulator (SSMI)]. The value of $\beta$ for spinone bosons was calculated microscopically in Ref 12 and turns out to be always negative. 
pseudo spin operators is redundant: the phase string appears twice in the calculation. Taking this point of view, we find it is more convenient and natural to employ the bosonic representation (see section III) for spin operators. And we choose to use the bosonization technique to evaluate correlations at $\left(h_{z}=h_{c}\right)$ and close to critical points $\left(h_{z}>h_{c}\right)$.

The bosonization scheme is a generic representation to address interacting bosons as well as Fermions in onedimension systems. It is particularly powerful when describing algebraically ordered quantum spin nematic liquids close to the critical points. And it also serves very well as a framework to study the equivalence between the interacting boson model in section III and the Fermion (free and interacting) models in section IV; in fact the dynamics of algebraically ordered nematic spin liquids is identical to Luttinger liquid ones. We are going to work in the continuum limit.

First we rewrite the Hamiltonian in Eq. (15) in terms of $c^{\dagger}(x), c(x)$ in the continuum limit ( we have chosen the lattice constant $a$ to be one and $x$ is dimensionless),

$$
\begin{aligned}
& \frac{H_{X X Z}}{\epsilon J_{e x}}=\int d x c^{\dagger}(x)\left(2 h_{c}-2 h_{z}-\frac{\hbar^{2}}{2 m} \partial_{x}^{2}\right) c(x) \\
& -4 \beta \int d x d x^{\prime} v_{B}\left(x-x^{\prime}\right) c^{\dagger}(x) c(x) c^{\dagger}\left(x^{\prime}\right) c\left(x^{\prime}\right) .
\end{aligned}
$$

Here $-4 \beta v_{B}\left(x-x^{\prime}\right)$ represents the short ranged two-body potential in the lattice model and in the continuum limit it is taken to be a $\delta$-function, i.e., $v_{B}\left(x-x^{\prime}\right)=\delta\left(x-x^{\prime}\right)$. The dimensionless mass of bosons is set to be $m=1 / 4$.

Following Ref 21, we introduce the following standard conjugating bosonic fields for the creation operator of bosons $c^{\dagger}(x)$ (See details in appendix D),

$$
\begin{aligned}
& c^{\dagger}(x)=\sqrt{\rho\left(h_{z}\right)+\frac{\partial_{x} \Theta(x)}{\pi}} e^{i \Phi(x)} \sum_{m=-\infty}^{+\infty} e^{2 m i \Theta(x)}(; 38) \\
& {\left[\partial_{x} \Theta(x), \Phi\left(x^{\prime}\right)\right]=i \pi \hbar \delta\left(x-x^{\prime}\right) .}
\end{aligned}
$$

And $\rho\left(h_{z}\right)$ has been given in Eq.(21) in section III.

Substituting this Ansatz into Eq.(14) and taking the long wave length limit, we find the following Hamiltonian in terms of $\Theta(x)$ and $\Phi(x)\left(h_{c}>h_{z}\right)$,

$$
\begin{aligned}
& \frac{H_{X X Z}}{\epsilon J_{e x}}=\left(2 h_{c}-2 h_{z}+\mu\right) \frac{1}{\pi} \int d x \partial_{x} \Theta(x)+ \\
& \int d x\left[\frac{1}{2 \pi^{2}} \frac{\partial \mu}{\partial \rho}\left(\partial_{x} \Theta(x)\right)^{2}+\frac{\hbar^{2}}{2 m} \rho\left(h_{z}\right)\left(\partial_{x} \Phi(x)\right)^{2}\right](40)
\end{aligned}
$$

For an unrenormalized theory, the bare value of $\mu\left(\beta, h_{z}\right)$ is taken to be $-8 \beta \rho\left(h_{z}\right)$ and $\partial \mu / \partial \rho=-8 \beta$. Since the theory is renormalizable at the long wave length limit, we expect that the low energy effective Hamiltonian should be of the same form as in the bare case except that quantities such as $\mu$ can be strongly renormalized by short wave length interactions. In fact this form of the effective Hamiltonian is suggested by the hydrodynamics of bosonic gases ${ }^{21.22}$, though the $\gamma$-dependence of the renormalized $\mu$ and $\partial \mu / \partial \rho$ can be obtained only when certain microscopics are taken into account.

Therefore, the effective Hamiltonian only depends on the density and the renormalized functional form of the chemical potential $\mu(\rho)$. And both the chemical potential and the compressibility $\partial \mu / \partial \rho$ are functions of the dimensionless interaction constant $\gamma\left(\beta, h_{z}\right)$ defined in Eq.(18). Following Eq.(21), one finds that

$$
\gamma\left(\beta, h_{z}\right)=\frac{4 \pi \beta}{\sqrt{h_{z}-h_{c}}}
$$

as a function of $h_{z}$.

In equilibrium, the first term in Eq. (40) which is linear in $\Theta(x)$ has to vanish because of the stability of the system. This sets the known relation between the chemical potential and external field, $2\left(h_{z}-h_{c}\right)=\mu(\rho)$ which has been discussed in the previous sections. For the weakly interacting systems, in the leading order of $\gamma$, the chemical potential takes the bare value of $-8 \beta \rho$; for the strongly interacting case as in the $1 \mathrm{D}$ dilute limit, $\mu$ is renormalized and discussions in section III show that $\mu$ is proportional to $2 \pi^{2} \rho^{2}$ in the leading order of $1 / \gamma$.

Taking this into account, we substitute into Eq. (40) the renormalized chemical potential $\mu$. We recast it in a more familiar form

$$
\frac{H_{X X Z}}{\epsilon J_{e x}}=\frac{\hbar}{2 \pi} \int d x\left[v_{N}\left(\partial_{x} \Theta(x)\right)^{2}+v_{J}\left(\partial_{x} \Phi(x)\right)^{2}\right] .
$$

Here

$$
v_{N}\left(\beta, h_{z}\right)=\frac{1}{\pi \hbar} \frac{\partial \mu(\rho)}{\partial \rho\left(h_{z}\right)}, v_{J}=\pi \hbar \frac{\rho\left(h_{z}\right)}{m} ;
$$

again $m=1 / 4$. An alternative way of calculating $v_{N}$ can be found in an early work ${ }^{21}$. $v_{J}$ here is taken to be independent of interactions as a result of Galilean invariance (approximate) in the continuum limit; in general when periodical or random potentials are present, $v_{J}$ could be renormalized away from this value.

To calculate $v_{N}$ and $v_{J}$, one has to take into account the Bethe Ansatz result for the chemical potential in Eq.(20). One obtains the following results for $v_{N}$,

$$
v_{N}=4 \pi \hbar \rho\left(h_{z}\right) \frac{(-4 \beta)^{4}}{\left[-4 \beta+2 \rho\left(h_{z}\right)\right]^{4}}
$$

Eq.(21) shows that both $v_{N}$ and $v_{J}$ vanish as $h_{z}$ approaches the critical field $h_{c}$. The discussions in Appendix $\mathrm{D}$ show that the nematic operator is algebraically correlated, and close to the critical point it takes the form

$$
\left\langle Q_{\alpha \beta}^{P_{x y}}(k) Q_{\alpha^{\prime} \beta^{\prime}}^{P_{x y}}(l)\right\rangle \sim \frac{T_{\alpha \beta} T_{\alpha^{\prime} \beta^{\prime}}(1-\rho) \rho}{(\rho|k-l|)^{1 / \eta}} .
$$


in the long-wave length limit, where the correlation exponent is determined by the value of $\eta$

$$
\eta\left(\beta, h_{z}\right)=2 \sqrt{\frac{v_{J}}{v_{N}}}=2\left[1+\frac{\sqrt{h_{z}-h_{c}}}{2 \pi \beta}\right]^{2} .
$$

In Fig.1 one can find more discussions on various phases in the bosonic representation and implications on spinone bosons in one-dimensional lattices.

The sound velocity of the Holstein-Primakov bosons corresponds to the spin-wave velocity in nematic states. Close to the critical points when $\rho\left(h_{z}\right)$ vanishes, $v_{N} \approx v_{J}$ and the spin wave velocity $v_{s}=\sqrt{v_{N} v_{J}}$ is

$$
v_{s}\left(h_{z}\right) \approx 4 \pi \hbar \rho\left(h_{z}\right)=4 \hbar \sqrt{h_{z}-h_{c}}+\ldots
$$

following Eq.(21).

Eq.(39) and Eq.(42) also define the Luttinger liquid dynamics of interacting Fermions ${ }^{23.24,25,26}$. In the standard formulation of the Luttinger liquid theory ${ }^{26}$, the left-moving and right moving Fermion operators are expressed in terms of bosonic fields of $\Theta(x)$ and $\Phi(x)$

$$
f_{L, R}^{\dagger}=\exp \left( \pm i q_{F} x\right) \exp (i \Theta(x) \pm i \Phi(x)) .
$$

The dynamics of $\Theta(x)$ and $\Phi(x)$ is given by

$$
\begin{aligned}
& H_{L . L .}=\frac{\hbar \sqrt{v_{N} v_{J}}}{2} \int d x\left[\frac{K}{\pi}\left(\partial_{x} \Phi(x)\right)^{2}+\frac{\pi}{K} \Pi^{2}(x)\right], \\
& {\left[\Pi(x), \Phi\left(x^{\prime}\right)\right]=i \hbar \delta\left(x-x^{\prime}\right) .}
\end{aligned}
$$

Here $\Pi(x)=\frac{1}{\pi} \partial \Theta(x)$. One can easily verify that Eq.(42) and Eq.(39) are identical to Eq.(49) and Eq.(150) if one identifies that

$$
\eta=2 K=2 \sqrt{\frac{v_{J}}{v_{N}}} .
$$

This intimate relation between 1D bosonic superfluid and Luttinger liquids was appreciated before ${ }^{21}$.

\section{CONCLUSIONS AND DISCUSSIONS}

In this article, we have studied the critical phenomenon in the field-driven quantum phase transitions between the nematic Mott state and spin singlet Mott state in onedimensional lattices. The critical states in one-dimension are in the universality class of impenetrable bosons or free Fermions. We have found that because of hardcore nature of magnons in one-dimension the critical exponent of magnetization is renormalized to be one-half of the value in three-dimensional lattices.
We have also calculated the nematic spin correlations using the Bosonization scheme and obtained Zeemanfield-dependent algebraically ordered nematic spin liquids. At critical points, the nematic spin correlation functions are identical to two-point correlations of onedimensional free Fermions with the Friedel oscillations completely suppressed. Close to critical points, the algebraically nematically ordered states are equivalent to Luttinger liquids of weakly attractive spinless Fermions or fluid of strongly repulsive bosons.

F.Z. thanks CASTU, Tsinghua University, Beijing for its hospitality during his stay in the summer of year 2004; he would like to acknowledge useful discussions with I. Affleck. This project is in part supported by NSF, P. R. China(Grant No. 10247002 and 10404015)(H.Z.) and in part supported by a grant from UBC and NSERC, Canada (F.Z.).

Note added: The transition described here might be considered as an analogue of commensurate- incommensurate transitions and is of quantum Pokrovsky- Talapov type 27 .

\section{APPENDIX A: NEMATIC TENSOR ORDER PARAMETER IN THE PSEUDO SPIN SUBSPACE}

Close to critical points, the Hilbert space is truncated into a two-level subspace at each site. Therefore we can define a pseudo-spin $1 / 2$ at each site, where $|\uparrow\rangle$ denotes the state $\left|S+2, S_{z}=S+2\right\rangle$ and $|\downarrow\rangle$ denotes the state $\left|S, S_{z}=S\right\rangle$. In this truncated subspace the nematic operator

$$
\hat{Q}_{\alpha \beta}=\psi_{\alpha}^{\dagger} \psi_{\beta}-\frac{1}{3} \delta_{\alpha \beta} \psi_{\gamma}^{\dagger} \psi_{\gamma},
$$

can be expressed in terms of pseudo-spin operators.

Consider the case of 2-particle per site. After some straightforward calculations, the matrix elements of the nematic operator can be obtained as

$$
\left\langle\uparrow\left|\hat{Q}_{\alpha \beta}\right| \uparrow\right\rangle=\left(\begin{array}{ccc}
\frac{1}{3} & i & 0 \\
-i & \frac{1}{3} & 0 \\
0 & 0 & -\frac{2}{3}
\end{array}\right),
$$

$$
\left\langle\uparrow\left|\hat{Q}_{\alpha \beta}\right| \downarrow\right\rangle=\frac{1}{\sqrt{3}}\left(\begin{array}{ccc}
1 & -i & 0 \\
-i & -1 & 0 \\
0 & 0 & 0
\end{array}\right)=\left\langle\downarrow\left|\hat{Q}_{\alpha \beta}\right| \uparrow\right\rangle^{*},
$$

and

$$
\left\langle\downarrow\left|\hat{Q}_{\alpha \beta}\right| \downarrow\right\rangle=0 .
$$

Therefore, for an arbitrary state $|\Omega\rangle$ in this subspace

$$
|\Omega\rangle=\cos \frac{\theta}{2} \exp \left(-i \frac{\phi}{2}\right)|\uparrow\rangle+\sin \frac{\theta}{2} \exp \left(i \frac{\phi}{2}\right)|\downarrow\rangle,
$$


the expectation value of the nematic operator is

$$
\begin{aligned}
\left\langle\Omega\left|\hat{Q}_{\alpha \beta}\right| \Omega\right\rangle & =\cos ^{2} \frac{\theta}{2}\left(\begin{array}{ccc}
\frac{1}{3} & i & 0 \\
-i & \frac{1}{3} & 0 \\
0 & 0 & -\frac{2}{3}
\end{array}\right) \\
& +\sin \theta \exp (i \phi) \frac{1}{2 \sqrt{3}}\left(\begin{array}{ccc}
1 & -i & 0 \\
-i & -1 & 0 \\
0 & 0 & 0
\end{array}\right) \\
+ & \sin \theta \exp (-i \phi) \frac{1}{2 \sqrt{3}}\left(\begin{array}{ccc}
1 & i & 0 \\
i & -1 & 0 \\
0 & 0 & 0
\end{array}\right)
\end{aligned}
$$

The first term in the right-hand side of above equation is contributed from the fully polarized state and should be projected away. The remained two terms give the expectation value of the projected nematic operator, which is

$$
\begin{aligned}
\left\langle\Omega\left|\hat{Q}_{\alpha \beta}^{P}\right| \Omega\right\rangle & =\sin \theta \exp (i \phi) \frac{1}{2 \sqrt{3}}\left(\begin{array}{ccc}
1 & -i & 0 \\
-i & -1 & 0 \\
0 & 0 & 0
\end{array}\right) \\
& +\sin \theta \exp (-i \phi) \frac{1}{2 \sqrt{3}}\left(\begin{array}{ccc}
1 & i & 0 \\
i & -1 & 0 \\
0 & 0 & 0
\end{array}\right)
\end{aligned}
$$

To express the projected nematic operator in terms of pseudo-spin operators, one should look for a suitable combination of the $\sigma$-matrices which yields the same expectation value as Eq.A3 , or Eq.A7. It is easily to verify that $\hat{Q}_{\alpha \beta}^{P}$ should be represented by the following operator in the pseudo-spin space,

$$
\frac{1}{2}\left(\sigma_{x}+\sigma_{y}\right) \Gamma_{\alpha \beta}+\frac{1}{2}\left(\sigma_{x}-i \sigma_{y}\right) \Gamma_{\alpha \beta}^{*},
$$

where

$$
\Gamma_{\alpha \beta}=\frac{1}{\sqrt{3}}\left(\begin{array}{ccc}
1 & -i & 0 \\
-i & -1 & 0 \\
0 & 0 & 0
\end{array}\right)
$$

\section{APPENDIX B: THE CHEMICAL POTENTIAL OF INTERACTING BOSONS IN ONE-DIMENSION}

The one-dimensional interacting bosons problem can be exactly solved by the Bethe Ansatz method. In this appendix we will briefly review the Betha Ansatz method used in Ref 19. Let $\left\{q_{i}\right\}(i=1, \cdots, N)$ be the quasimomenta of the $N$ bosons, the Bethe Ansatz solution indicates that all the allowed values of $q_{i}$ 's should satisfy the following set of equations:

$$
(-1)^{N-1} e^{-i q_{j} L}=\exp \left(i \sum_{i=1}^{N} \theta\left(q_{i}-q_{j}\right)\right),
$$

where $\theta_{i, j}$ is the phase shift. In our case, one finds that

$$
\theta\left(q_{i}-q_{j}\right)=-2 \arctan \frac{q_{i}-q_{j}}{-4 \beta}
$$

Dividing two successive equations and equating exponents, one obtains

$$
\left(q_{i+1}-q_{i}\right) L=\sum_{j=1}^{N}\left(\theta_{j, i}-\theta_{j, i+1}\right)+2 \pi I_{i}
$$

where a set of integers $I_{i}(i=1, \cdots, N)$ are the quantum numbers for this system, and they are all equal to one for the ground state. Taking the continuum limit of Eq.(B3), one has

$$
-8 \beta \int_{-\Lambda}^{\Lambda} d p \frac{n(p)}{16 \beta^{2}+(p-q)^{2}}=2 \pi n(q)-1
$$

where $\Lambda$ is the cut-off momentum which should be determined self-consistently. Here $n(q)$ is the distribution function of quasi-momentum, which satisfies the constraint

$$
\int_{-\Lambda}^{\Lambda} d q n(q)=\rho
$$

And the ground state energy is given by

$$
E_{0}=\frac{N}{\rho} \int_{-\Lambda}^{\Lambda} n(q) \frac{q^{2}}{2 m} d q
$$

Now rescale all qualities in terms of the cut-off momentum $\Lambda$, i.e. $-4 \beta=\Lambda \lambda, p=\Lambda y$ and $q=\Lambda x$, and denote $n(q)$ as $g(x)$. The above three equations take the following form

$$
1+2 \lambda \int_{-1}^{1} \frac{g(y)}{\lambda^{2}+(y-x)^{2}} d y=2 \pi g(y)
$$

$$
\gamma \int_{-1}^{1} g(x) d x=\lambda
$$

and

$$
E_{0}=\frac{1}{2 m} N \rho^{2} \frac{\gamma^{3}}{\lambda^{3}} \int_{-1}^{1} g(x) x^{2} d x
$$

In the limit of large $\gamma, \lambda$ is large compared to $|x-y|$, one can then neglect the $x$ dependence of the integrand in Eq.(B7); and $g(x)$ turns out to be a constant. The integral equation can be solved approximately and the result is

$$
g=\frac{\lambda}{2 \pi \lambda-4} .
$$


Substituting it back to Eq.(B8) one obtains the relation between $\lambda$ and $\gamma$ as

$$
\lambda=\frac{\gamma+2}{\pi} .
$$

And finally, using Eq. (B9) one can derive the ground state energy

$$
E_{0}=N \rho^{2} \frac{\pi^{2}}{6 m}\left(\frac{\gamma}{\gamma+2}\right)^{2}
$$

Consequently, a relation between chemical potentials and particle densities is established in this limit,

$$
\mu=\frac{\partial E_{0}}{\partial N}=\frac{\pi^{2}}{6 m} \frac{3 \gamma^{3}+2 \gamma^{2}}{(\gamma+2)^{3}} \rho^{2} .
$$

\section{APPENDIX C: THE JORDAN-WIGNER FERMION REPRESENTATION}

\section{The effective Hamiltonian for Jordan-Wigner Fermions in the XXZ model}

In this appendix we will briefly review the JordanWigner Fermionic representation of the XXZ model, the Hamiltonian of which is

$$
\begin{gathered}
\frac{H_{X X Z}}{\epsilon J_{\mathrm{ex}}}=-2 \sum_{<k l>}\left(\sigma_{k}^{+} \sigma_{l}^{-}+\sigma_{k}^{-} \sigma_{l}^{+}\right) \\
-(\beta+1) \sum_{<k l>} \sigma_{k z} \sigma_{l z}-h_{z} \sum_{k} \sigma_{z} .
\end{gathered}
$$

Following the Jordan-Wigner transformation Eq.(26), the spin flipping term becomes

$$
\begin{aligned}
& \sigma_{k}^{+} \sigma_{k+1}^{-}=e^{i \pi \sum_{l=1}^{k-1} f_{l}^{\dagger} f_{l}} f_{k}^{\dagger} f_{k+1} e^{-i \pi \sum_{l=1}^{k} f_{l}^{\dagger} f_{l}}=f_{k}^{\dagger} e^{-i \pi f_{k}^{\dagger} f_{k}} f_{k+1} \\
& =f_{k}^{\dagger}\left(1-2 f_{k}^{\dagger} f_{k}\right) f_{k+1}=f_{k}^{\dagger} f_{k+1},
\end{aligned}
$$

and similarly,

$$
\sigma_{k+1}^{+} \sigma_{k}^{-}=f_{k+1}^{\dagger} f_{k} .
$$

These yield a kinetic term of these Fermions. Neglecting a constant term, the second term in Eq.C1 turns out to be

$$
\sum_{k l} \sigma_{k z} \sigma_{l z}=4 \sum_{k l} f_{k}^{\dagger} f_{k} f_{l}^{\dagger} f_{l}-4 \sum_{k} f_{k}^{\dagger} f_{k} .
$$

Thus one arrives at the Jordan-Wigner Fermion representation of the XXZ model as shown in Eq.(27).

Finally, due to the Pauli exclusive principle one can treat the interacting terms as follows

$$
\begin{aligned}
& \sum_{k} f_{k}^{\dagger} f_{k+1}^{\dagger} f_{k} f_{k+1}=\sum_{k}\left(f_{k}^{\dagger} f_{k+1}^{\dagger} f_{k} f_{k+1}-f_{k}^{\dagger} f_{k}^{\dagger} f_{k} f_{k}\right) \\
& =\sum_{q, q_{1}, q_{2}} f_{q_{1}-q}^{\dagger} f_{q_{2}+q}^{\dagger} f_{q_{1}} f_{q_{2}}(\cos q-1),
\end{aligned}
$$

and this results in Eq.(29).

\section{Calculation of Eq. (36)}

In the continuum limit, $\sigma^{ \pm}$can be written in the following form

$$
\begin{aligned}
\sigma^{+} & =\exp \left(-i q_{F} x-i \Theta(x)\right) f^{\dagger}(x) \\
\sigma^{-} & =f(x) \exp \left(i q_{F} x+i \Theta(x)\right)
\end{aligned}
$$

Taking into account the expression for the left- and rightmovers in Eq.(48), one obtains

$$
\begin{aligned}
& \sigma^{+} \sim \exp (i \Phi(x))+\exp \left(-i 2 q_{F} x-i \Phi(x)\right) \\
& \sigma^{-} \sim \exp (-i \Phi(x))+\exp \left(i 2 q_{F} x+i \Phi(x)\right) .
\end{aligned}
$$

Therefore the leading contribution to the lowest harmonics in the correlation function is

$$
<\sigma^{+} \sigma^{-}>\sim<\exp (i \Phi(x)-i \Phi(0))>.
$$

Calculation of this correlator is the same as those in Appendix D. For free Fermions, one obtains the result in Eq.(36).

\section{APPENDIX D: BOSONIZATION AND NEMATIC SPIN CORRELATIONS}

In this appendix we briefly review the bosonization procedure for one-dimensional interacting bosonic system $^{21}$. First we express the bosonic operator as

$$
c^{\dagger}(x)=\sqrt{\rho_{T}(x)} e^{i \Phi(x)}
$$

The mean ground state density is denoted by $\rho_{0}$, we introduce a field $\Pi(x)$ to account for the long-wave length $\left(>\rho_{0}^{-1}\right)$ density fluctuations. Furthermore in order to take the discreteness of particles into account, we introduce an auxiliary field $\Theta(x)$ which is related to $\Pi(x)$ via

$$
\frac{1}{\pi} \partial_{x} \Theta(x)=\rho_{0}+\Pi(x),
$$

where $\Theta$ increases monotonically by $\pi$ each time when $x$ passes the location of a particle. Therefore the total density can be expressed as

$$
\rho_{T}(x)=\frac{1}{\pi} \partial_{x} \Theta(x) \sum_{n=-\infty}^{+\infty} \delta(\Theta(x)-n \pi),
$$

where the discrete character is incorporated. Since the square root of a $\delta$ function is also proportional to a $\delta$ function, it yields that

$$
\begin{aligned}
c^{\dagger}(x) & \sim \sqrt{\rho_{0}+\Pi(x)} \sum_{n=-\infty}^{+\infty} \delta(\Theta(x)-n \pi) e^{i \Phi(x)} \\
& =\sqrt{\rho_{0}+\Pi(x)} \sum_{m=-\infty}^{+\infty} e^{2 m i \Theta(x)} e^{i \Phi(x)}
\end{aligned}
$$


where the second equality follows from the Poison's summation identity

$$
\sum_{n=-\infty}^{+\infty} f(n)=\sum_{m=-\infty}^{+\infty} \int_{-\infty}^{\infty} d z f(z) e^{2 m \pi i z}
$$

It can be verified that the field $\Theta$ and $\Phi$ satisfy the commutative relation

$$
\left[\partial_{x} \Theta(x), \Phi\left(x^{\prime}\right)\right]=i \pi \hbar \delta\left(x-x^{\prime}\right) .
$$

With these expressions, the effective Luttinger Hamiltonian can be read as

$$
H_{\text {eff }}=\frac{\hbar}{2 \pi} \int_{0}^{L} d x\left[v_{J}\left(\partial_{x} \Phi(x)\right)^{2}+v_{N}\left(\partial_{x} \Theta(x)\right)^{2}\right] .
$$

Here we have introduced two phenomenological parameters $v_{J}$ and $v_{N} . v_{J}$ is the phase stiffness which equals to $\hbar \pi \rho / m$ in a uniform system, and $v_{N}$ is the density stiffness which is defined as

$$
v_{N}=\frac{1}{\pi \hbar}\left(\frac{\partial \mu}{\partial \rho}\right)
$$

These parameters should be determined by microscopic calculations. For boson gases with delta-like interactions, these parameters can be obtained from the Bethe Ansatz solutions.

Furthermore we can express the correlation of nematic operator in terms of correlators of Holstein-Primakov bosons,

$$
\begin{aligned}
& \left\langle Q_{\alpha \beta}^{P_{x y}}(k) Q_{\alpha^{\prime} \beta^{\prime}}^{P_{x y}}(l)\right\rangle=T_{\alpha \beta} T_{\beta^{\prime} \alpha^{\prime}}^{\dagger} \\
& \left\langle c^{\dagger}(k) \sqrt{1-c^{\dagger}(k) c(k)} \sqrt{\left.1-c^{\dagger}(l) c(l) c^{\dagger}(l)\right\rangle+h . c .}\right. \\
& \simeq T_{\alpha \beta} T_{\beta^{\prime} \alpha^{\prime}}^{\dagger}(1-\rho)\left\langle c^{\dagger}(k) c(l)\right\rangle+h . c .
\end{aligned}
$$

The correlation function for the Holstein-Primakov bosons can be computed using the effective Luttinger Hamiltonian,

$$
\begin{aligned}
& \left\langle c^{\dagger}(x) c(0)\right\rangle=\rho\left[\frac{1}{\rho|x|}\right]^{1 /(2 K)}\left\{b_{0}\right. \\
& \left.+\sum_{m=1}^{+\infty} b_{m}\left(\frac{1}{\rho|x|}\right)^{2 m^{2} K} \cos (2 \pi m \rho x)\right\} .
\end{aligned}
$$

Here

$$
\eta=2 K=2 \sqrt{\frac{v_{J}}{v_{N}}}
$$

is a universal parameter only depending on long-wave length physics, while the $b_{m}$ 's depend on the microscopic details.
1 For general discussions on fluctuations, please see this and Ref 2, 34. S. K. Ma, Modern theory of critical phenomena (Benjamin Cummings, Reading, 1976).

2 P. M. Chaikin and T. C. Lubensky, Principles of condensed matter physics (Cambridge University Press, 1995).

3 J. Zinn-Justin, Quantum field theory and critical phenomena(Oxford University Press, Oxford, 1993).

4 S. Sachdev, Quantum phase transitions (Cambridge Press, 1999).

${ }^{5}$ F. Zhou, Phys. Rev. Lett. 87, 080401-1 (2001); F. Zhou, Int. Jour. Mod. Phys. B 17 No. 14, 2643-2698 (2003).

6 E. Demler and F. Zhou, Phys. Rev. Lett. 88, 163001-1 (2002).

7 F. Zhou and M. Snoek, Annals of Physics 308, 692 (2003).

${ }^{8}$ Fei Zhou, Euro. Phys. Lett. 63(4), 505 (2003).

9 A. Imambekov, M. Lukin and E. Demler, Phys. Rev. A68, 063602 (2003).

10 M. Snoek and F. Zhou, Phys. Rev. B 69, 094410 (2004).

11 A. Imambekov, M. Lukin and E. Demler, Phys. Rev. Lett. 93, 120405 (2004).

12 F. Zhou, M. Snoek, J. Wiemer and I. Affleck, Phy. Rev. B 70, 184434 (2004)

13 I. Affleck, Phys. Rev. B 43, 3215 (1991);

${ }^{14}$ K. Nomura and T. Sakai, Phys. Rev. B 44, 5092 (1991);
T. Sakai and M. Takahashi, Phys. Rev. B43, 13383 (1991).

15 E. Sorensen and I. Affleck, Phys. Rev. Lett.71,1633 (1993).

16 A. Tsvelik, Phys. Rev. B 42, 10499 (1990).

17 See for instance recent works: P. R. Hammar, M. B. Stone, D. H. Recih, C. Broholm, P. J. Gibson, M. M. Turnbull, C. P. Landee, and M. Oshikawa, Phys. Rev. B 591008 (1999); I. Affleck and M. Oshikawa, Phys. Rev. B 60, 1038 (1999).

18 M. Girardeau, J. Math. Phys. 1, 516 (1960).

19 E. H. Lieb and W. Liniger, Phys. Rev. 130, 1605 (1965); E. H. Lieb, J. Math. Phys. (N.Y.) 6, 304 (1965).

20 C. N. Yang and C. P. Yang, Phys. Rev. 150 1, 321 (1966).

21 F. D. M. Haldane, Phys. Rev. Lett. 47, 1840 (1981); See also F. D. M. Haldane, J. Phys. C 14, 2585 (1981).

22 P. Nozieres and D. Pines, The theory of quantum liquids, Vol. II: Superfluid Bose Liquids (Addison-Wesley publishing Co., 1990).

23 S. Tomonaga, Prog. Theor. Phys. 5, 544 (1950).

24 J. M. Luttinger, J. Math. Phys. 4, 1154 (1963).

25 D. C. Mattis and E. H. Lieb, J. Math. Phys. 6, 304 (1965).

26 A. Luther and I. Peschel, Phys. Rev. B9, 2911 (1974); A. Luther and V. J. Emery, Phys. Rev. Lett. 33, 589 (1974).

27 V. L. Pokrovsky and A. L. Talapov, Phys. Rev. Lett. 42, 65 (1979). 